\title{
OMNIDIRECTIONAL PERCEPTION FOR LIGHTWEIGHT UAVS USING A CONTINUOUSLY ROTATING 3D LASER SCANNER
}

\author{
David Droeschel, Michael Schreiber, and Sven Behnke
}

Autonomous Intelligent Systems Group, Computer Science Institute VI, University of Bonn, Germany http://www.ais.uni-bonn.de; droeschel@ais.uni-bonn.de

KEY WORDS: UAVs, 3D Laser Scanner, Scan Registration, Obstacle Avoidance

\begin{abstract}
:
Many popular unmanned aerial vehicles (UAV) are restricted in their size and weight, making the design of sensory systems for these robots challenging. We designed a small and lightweight continuously rotating 3D laser scanner-allowing for environment perception in a range of $30 \mathrm{~m}$ in almost all directions. This sensor it well suited for applications such as 3D obstacle detection, 6D motion estimation, localization, and mapping. We aggregate the distance measurements in a robot-centric grid-based map. To estimate the motion of our multicopter, we register 3D laser scans towards this local map. In experiments, we compare the laser-based ego-motion estimate with ground-truth from a motion capture system. Overall, we can build an accurate 3D obstacle map and can estimate the vehicle's trajectory by 3D scan registration.
\end{abstract}

\section{INTRODUCTION}

In recent years, lightweight unmanned aerial vehicles (UAV) such as quadrotors attracted much attention in the field of aerial robotics, because of their relatively low cost, ease of control, and compatibility with everyday indoor and outdoor environments. The size and weight limitations of such platforms, however, pose a problem for the design of their sensory systems. Most of today's lightweight UAVs are equipped with ultra sound distance sensors and air pressure sensors for estimating hight above the ground, inertial sensors for estimating attitude, magnetometers for estimating the heading direction, and cameras, e.g. for estimating visual odometry. While these small and lightweight sensors provide valuable information, they do not suffice for obstacle avoidance and navigation. Only few systems (Tomić et al., 2012, Grzonka et al., 2009, Bachrach et al., 2009, Shen et al., 2011) are equipped with 2D laser range finders (LRF) that measure distances in a plane around the UAV.

2D laser range sensors are widely used for mobile robots navigating on flat ground-due to their accurate distance measurements even in bad lighting conditions and their large field-ofview $(\mathrm{FoV})$. For robots acting in $3 \mathrm{D}$ environments or driving on more difficult terrain, three-dimensional laser scanning sensors are popular. For instance, many autonomous cars perceive obstacles by means of a rotating laser scanner with a $360^{\circ}$ horizontal FoV, allowing for detection of obstacles in all directions (Urmson et al., 2008, Montemerlo et al., 2008). Up to now, such 3D laser scanners are rarely used on lightweight UAVs, due to their payload limitations.

In order to enable navigation in difficult $3 \mathrm{D}$ environments for lightweight UAVs, we designed a continuously rotating 3D laser scanner that is minimalistic in terms of size and weight and measures distances of up to $30 \mathrm{~m}$ in almost all directions. Fig. 1 shows the sensor mounted on our multicopter.

We use the laser scanner to perceive obstacles around the robot by aggregating the distance measurements in a robot-centric $3 \mathrm{D}$ map. We estimate the $6 \mathrm{D}$ robot motion by registering 3D laser scans with this local map.

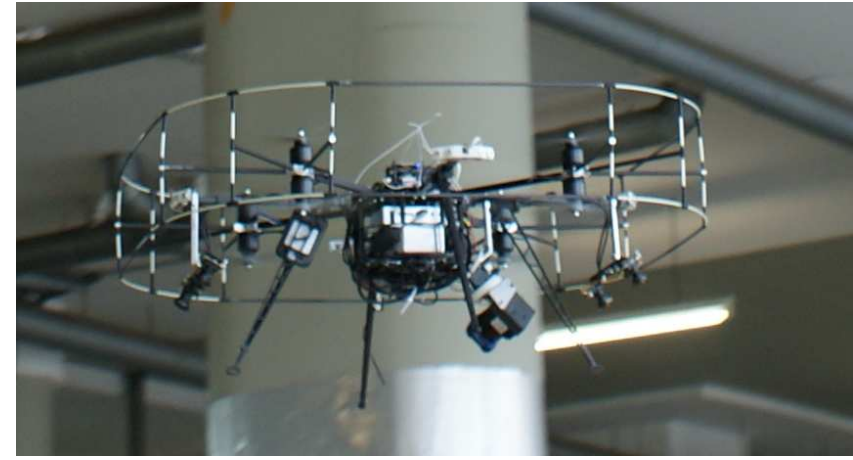

Figure 1: The 3D laser scanner mounted on our multicopter.

\section{RELATED WORK}

The use of UAVs in recent robotics research varies largely in the level of autonomy-ranging from basic hovering and position holding (Bouabdallah et al., 2004) over trajectory tracking and waypoint navigation (Puls et al., 2009) to fully autonomous navigation (Grzonka et al., 2012). Similarly, the complexity of environments where UAVs fly ranges from flight arenas instrumented with motion capture systems and external computing, over outdoor flights in open spaces where GPS is available, to indoor flights in restricted spaces. Limiting factors for increasing the level of autonomy and/or the complexity of environments for lightweight UAVs are onboard sensing and onboard processing power.

Particularly important for fully autonomous operation of UAVs are the abilities to perceive obstacles and to avoid collisions. Most autonomous UAVs, however, cannot adequately perceive their surroundings and, hence, cannot avoid all collisions. Instead, collision avoidance is often restricted to the two-dimensional measurement plane of laser range finders (Grzonka et al., 2012) or to the limited field-of-view of forward-facing cameras (Mori and Scherer, 2013, Ross et al., 2013). Most often, collision avoidance is neglected altogether, e.g., by flying in a certain height when autonomously flying between waypoints.

One way to extend the FoV for obstacle detection is to combine multiple sensors. Tomić et al., for example, present an autonomous UAV that perceives its environments using a stereo camera 


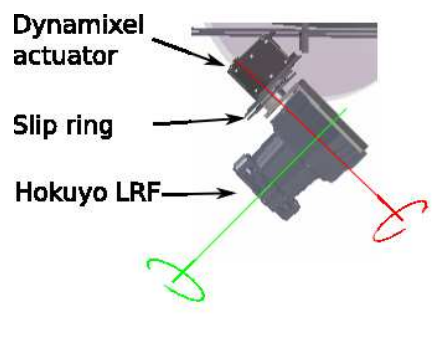

(a)

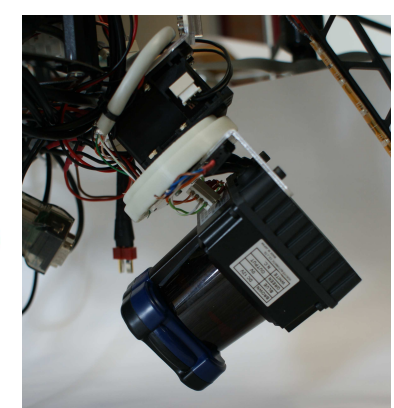

(b)
Figure 2: (a) CAD drawing of the continuously rotating laser scanner with the two rotation axes. The Hokuyo 2D LRF is mounted on a bearing and rotated around the red axis. (b) A photo of the assembled laser scanner.

pair mounted in forward direction and a 2D laser range scanner mounted horizontally (Tomić et al., 2012). Still, their perceptual field does not include the space below, above, and behind the robot. Most similar to our work is the work of Scherer, Cover et al. (Scherer et al., 2012, Cover et al., 2013). The authors describe a system that is used to autonomously explore rivers using visual localization and laser-based 3D obstacle perception. Similar to their approach, we aim at perceiving as much of the surroundings as possible in order to obtain almost omnidirectional obstacle detection.

\section{SENSOR SETUP}

Our continuously rotating 3D laser scanner consists of a Hokuyo UTM-30LX-EW 2D laser range finder (LRF) which is rotated by a Dynamixel MX-28 servo actuator to gain a three-dimensional FoV. As shown in Fig. 2, the scanning plane is parallel to the axis of rotation, but the heading direction of the scanner is twisted slightly away from the direction of the axis-in order to enlarge its field-of view. The 2D LRF is electrically connected by a slip ring, allowing for continuous rotation of the sensor. The axis of rotation is pitched downward by $45^{\circ}$ in forward direction, which places the core of the robot upwards behind the sensor. Hence, the sensor can measure in all directions, except for a conical blind spot pointing upwards behind the robot.

The 2D laser scanner has a size of $62 \times 62 \times 87.5 \mathrm{~mm}$ and a weight of $210 \mathrm{~g}$. Together with the actuator $(72 \mathrm{~g})$ and and the slip ring, the total weight of the 3D scanner is approximately $400 \mathrm{~g}$.

The Hokuyo LRF has an apex angle of $270^{\circ}$ and an angular resolution of $0.25^{\circ}$, resulting in 1080 distance measurements per $2 \mathrm{D}$ scan, called a scan line. The Dynamixel actuator rotates the 2D $\mathrm{LRF}$ at one rotation per second, resulting in 40 scan lines and 43,200 distance measurements per full rotation. Slower rotation is possible if a higher angular resolution is desired. For our setup, a half rotation leads to a full 3D scan of most of the environment. Hence, we can acquire 3D scans with up to 21,600 points with $2 \mathrm{~Hz}$.

\subsection{Calculating 3D Point Clouds}

In order to calculate a $3 \mathrm{D}$ point cloud from the scan lines originated by the LRF, a 3D point $P_{l}$ in the LRF's coordinate frame is transformed to $P_{b}$ in the UAV's base coordinate frame by

$$
P_{b}=T_{l} T_{r} T_{c} P_{l}
$$

Here, $T_{l}$ is a static transformation between the UAV's base frame and the link where the $3 \mathrm{D}$ laser scanner is mounted and $T_{c}$ is the static transformation between the $2 \mathrm{D} \mathrm{LRF}$ and the bearing. $T_{r}$ is a non-static transformation that takes the bearing's orientation into account. It's rotational parts are generated from the filtered encoder positions reported by the Dynamixel actuator. Fig. 3 shows a resulting scan of an indoor environment.

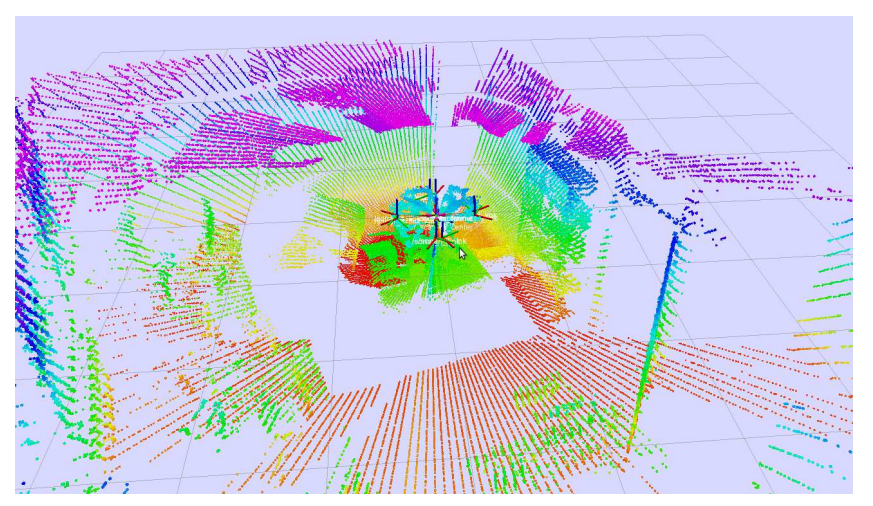

Figure 3: A 3D scan of an indoor environment acquired with our continuously rotating laser scanner.

\subsection{Orientation Filter}

Calculating a 3D point cloud from the distance measurements requires knowledge of the rotation angle of the scanner at the time the distance measurements are made. This angle is measured by the encoder of the Dynamixel actuator, but angle measurements are not exactly synchronized to distance measurements. Varying communication latencies with both sensors cause variance between actual scan line angle and angular measurements. To cope with this effect, we estimate the rotation angle of the laser scanner around the Dynamixel axis based on the measured encoder angles, assuming a constant rotational velocity. Fig. 4 shows the effect resulting from the varying communication latencies and the evenly spaced scans resulting from our angle estimate.

\subsection{Multi-Echo Detection}

The Hokuyo UTM-30LX-EW is able to measure up to three echoes of a single emitted light pulse. The number of echoes for a light pulse depends on the surface of the measured objects, i.e., shape and reflectivity. For example, transparent materials, vegetation or edges of buildings often yield more than one echo. Often, the second echo comes from a structure in the original pulse direction, behind a partial occluder, which means that it can be treated as an additional distance measurement.

\subsection{Self-Filter}

The laser range finder measures also points on the robot. These points are excluded from further processing by checking the robotcentric point coordinates against the bounding box of our robot.

\section{OBSTACLE MAP}

We accumulate the remaining distance measurements of in an egocentric 3D grid-based map which is centered around the robot. For each distance measurement and the corresponding 3D point, the individual cell of the map is marked as occupied. An exemplary map from a simulated environment is shown in Fig. 5. The map is used by our obstacle avoidance control, which uses a predictive potential field method to avoiding occupied cells (Nieuwenhuisen et al., 2013). 
(a)

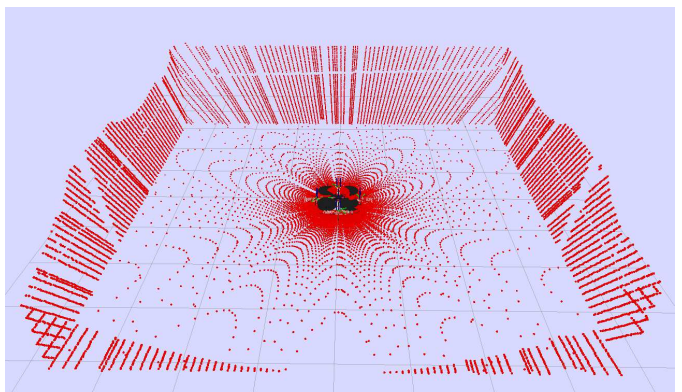

(b)

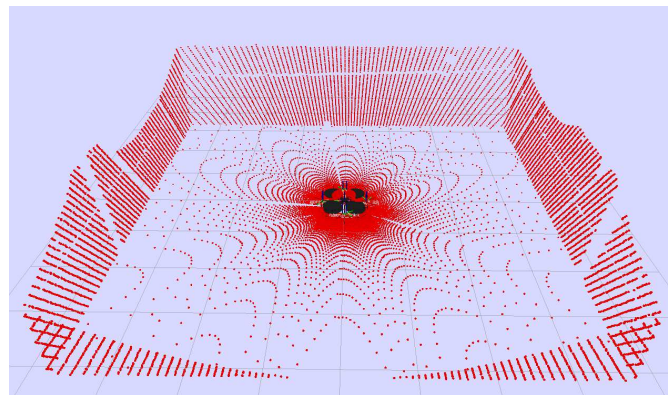

Figure 4: 3D laser scan from a simulated environment. (a) The effects resulting from varying communication latencies. (b) After filtering the encoder position assuming constant rotational velocity.

Along with the occupancy information, each cell also maintains its 3D scan points. These 3D points can be used for point-based scan processing, for example $3 \mathrm{D}$ scan registration.

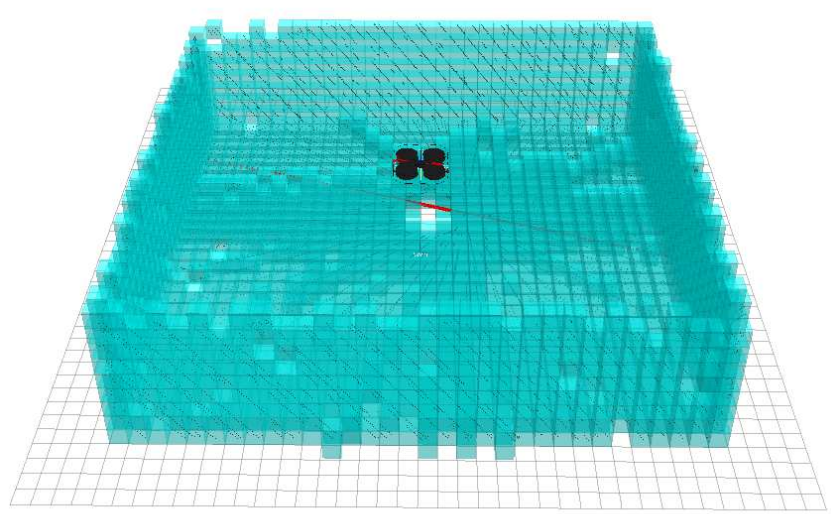

Figure 5: An exemplary grid-based map of a simulated environment.

We aim for efficient map management for translation and rotation. Therefore, individual grid cells are stored in a circular buffer to allow shifting of elements in constant time. We interlace multiple circular buffers to obtain a map with three dimensions. The length of the circular buffers depends on the resolution and the size of the map. In case of a translation of the UAV, the circular buffers are shifted whenever necessary to maintain the egocentric property of the map. In case of a translation equal or larger than the cell size, the circular buffers for respective dimensions are shifted. For sub-cell-length translations, the translational parts are accumulated and shifted if they exceed the length of a cell.

Since we store 3D points for every cell for point-based processing, single points are transformed in the cell's local coordinate

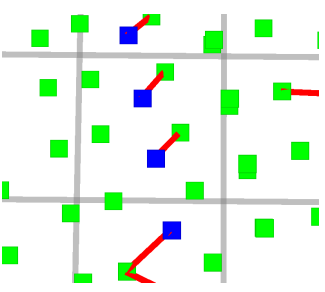

(a)

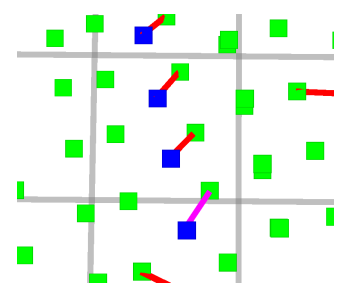

(b)
Figure 6: Assigning point correspondences. (a) For every point of a 3D scan (blue), a corresponding map point (green) is initially assigned from the cell's point list (red line). (b) If the distance to neighboring cells is smaller than the distance to the initial assignment, closer points might be found in the neighboring cell (magenta line).

frame when adding, and back to the map's coordinate frame when accessing. Every cell in the map stores a list of 3D points from the current and previous 3D scans. This list is also implemented by a fixed-sized circular buffer.

Since rotating the map would necessitate to shuffle all cells, our map is oriented independent to the UAV's orientation. We maintain the orientation between the map and the UAV and use it to rotate measurements when accessing the map.

\section{SCAN REGISTRATION}

We register 3D scans of the sensor to estimate the robot's egomotion. A complete 3D scan is aligned to the map by the Iterative Closest Point (ICP) algorithm. The ICP algorithm estimates a transformation between two point clouds, describing the displacement between them. The scan registration is triggered after acquiring a full 3D scan (i.e., a half rotation).

\subsection{Data Association}

When using the ICP algorithm for scan registration, corresponding points between the model and the current point cloud are assigned, usually by building a space-partitioned data structure from the model point cloud. In contrast, we utilize our gridbased data structure for efficient correspondence assignment. Every point from a newly acquired $3 \mathrm{D}$ scan is directly assigned to a map cell in the map in constant time. The closest point in terms of the Euclidean distance from the point list of this cell is initially assigned as corresponding point.

As illustrated in Fig. 6, points in the neighboring cells might be closer to the measured point than the initially assigned point. Consequently, we extend the search to neighboring cells, if the distance to the initial assignment is larger than the distance to the border of a neighboring cell.

\subsection{Transformation Estimation}

With $N$ assigned corresponding point pairs $\left(m_{i}, d_{i}\right)$, we determine the displacement between the points of a scan $d_{i}$ and the map points $m_{i}$ by finding a rigid transformation $T$ that minimizes

$$
E(\mathbf{T})=\sum_{i=1}^{N}\left\|\mathbf{m}_{i}-\mathbf{T} d_{i}\right\|^{2}
$$

by a closed-form singular value decomposition (SVD) algorithm (Besl and McKay, 1992). 


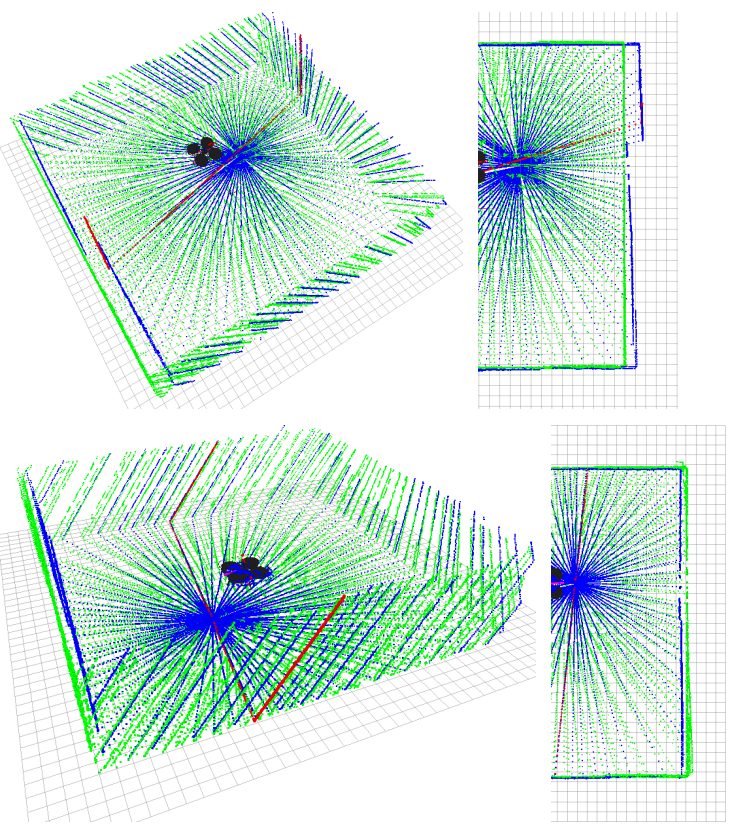

Figure 7: Sensor movement during scan acquisition yields distorted 3D scans (top). We deskew the scan based on the motion estimate (bottom).

In each ICP iteration, correspondences are re-assigned, the transformation that aligns these best is applied to the scan, and the following termination criteria are checked:

- $E(\mathbf{T})$ is smaller than a given threshold $t h_{r}$,

- the difference between $T_{k}$ and $T_{k-1}$ is smaller than $t h_{\epsilon}$, or

- the number of iterations exceeds $t h_{i}$,

where $T_{k}$ and and $T_{k-1}$ are the estimated transformations from the current and the previous iteration, respectively. Thresholds $t h_{r}, t h_{i}$ and $t h_{\epsilon}$ are manually determined. In our experiments, $t h_{r}=1 \mathrm{~cm}, t h_{\epsilon}=0.001 \mathrm{~cm}$ and $t h_{i}=5$ showed best results. If one of the criteria is met, the estimated transformation is applied to the scan lines of the 3D scan, undistorting it as described in the next paragraph.

\subsection{Scan Undistortion}

Since movement of the sensor during acquisition leads to a distortion of the 3D scan, we use the estimated transformation to deskew it. The rotational and translational parts of the displacement are distributed over the acquisition time by assuming constant velocities, i.e. fractional displacements are applied for consecutive scan lines. For an acquired 3D scan, the transformation that has been estimated for the previous scan is used as an initial guess for deskewing prior to registration. After convergence of the ICP algorithm, the scan is deskewed with the estimated transformation. Fig. 7 illustrates the scan undistortion.

\section{EXPERIMENTS}

In order to evaluate the scan registration, we have conducted experiments in an indoor motion capture (MoCap) system shown in Fig. 9a. The MoCap system provides accurate pose information of the UAV at high frame rates $(100 \mathrm{~Hz})$ but is restricted in to a capture volume of approximately $2 \times 2 \times 3 \mathrm{~m}$. We compare laserbased odometry from registering $3 \mathrm{D}$ scans with the pose measured by the MoCap system. Fig. 9b visually compares the estimated laser-based trajectory to the ground-truth data from MoCap system. One can observe that the trajectory from the motion capture system is denser than the estimated trajectory, since the frame rate is higher. Fig. 10 shows the resulting translational and rotational errors.

In terms of run time, the most computational expensive step in the scan registration is the correspondence assignment, which has to be carried out in every iteration. Depending on the run time of the correspondence assignment, the parameter $t h_{i}$ mentioned in Section 5.2 can be used to control the maximum run time of the entire scan registration. Table 1 shows the average run times of the correspondence assignment for the aforementioned indoor experiment on a single core of an Intel Core2 Duo $(2.4 \mathrm{GHz})$ processor.

Table 1: Runtime of correspondence assignment for an indoor environment on a single core of an Intel Core2 Duo $(2.4 \mathrm{GHz})$ processor.

\begin{tabular}{|c|l|l|}
\hline $\begin{array}{c}\text { Resolution } \\
\text { (cells / meter) }\end{array}$ & $\begin{array}{l}\text { Average } \\
\text { runtime (s) }\end{array}$ & $\begin{array}{l}\text { Standard } \\
\text { deviation }\end{array}$ \\
\hline 2 & 0.0980927 & 0.0677847379 \\
4 & 0.1014915909 & 0.0632963261 \\
8 & 0.137749037 & 0.0991583993 \\
16 & 2.484522 & 1.6934523199 \\
\hline
\end{tabular}

Note that these run times depend on the number of cell accesses, the maximum length of the point list in each cell, the density of the point cloud and therefore strongly on the scene. In this experiment we used a resolution of 2 cells / meter and achieve an average run time of $0.418017 \mathrm{~s}$ for the complete scan registration. A point list length of 250 was used in the experiment.

Resulting point clouds of the sensor in an outdoor environment are shown in Fig. 11. A point cloud from a relative small elongated object is shown in figure Fig. 8, demonstrating that the sensor is able to perceive such small obstacles. Besides that, we have also conducted experiments with dynamical obstacles, showing that the UAV is able to omnidirectional perceive obstacles and to react on them (Nieuwenhuisen et al., 2013).

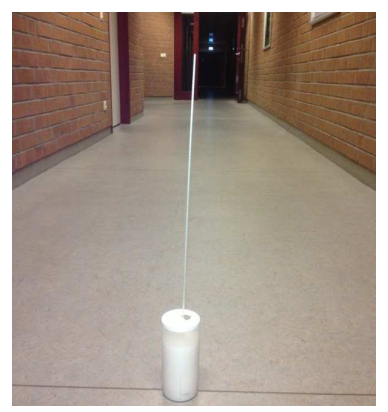

(a)

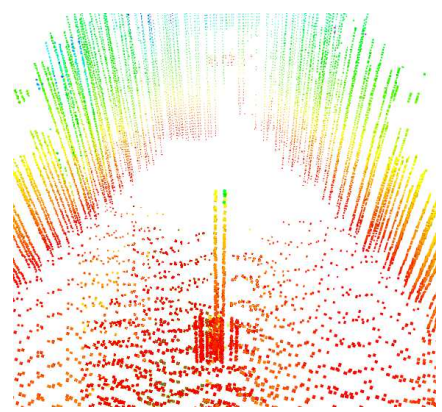

(b)
Figure 8: Perception of small obstacles: (a) rod with $2.5 \mathrm{~mm}$ diameter; (b) resulting 3D point cloud (pixel color encodes height above ground plane). 
(a)
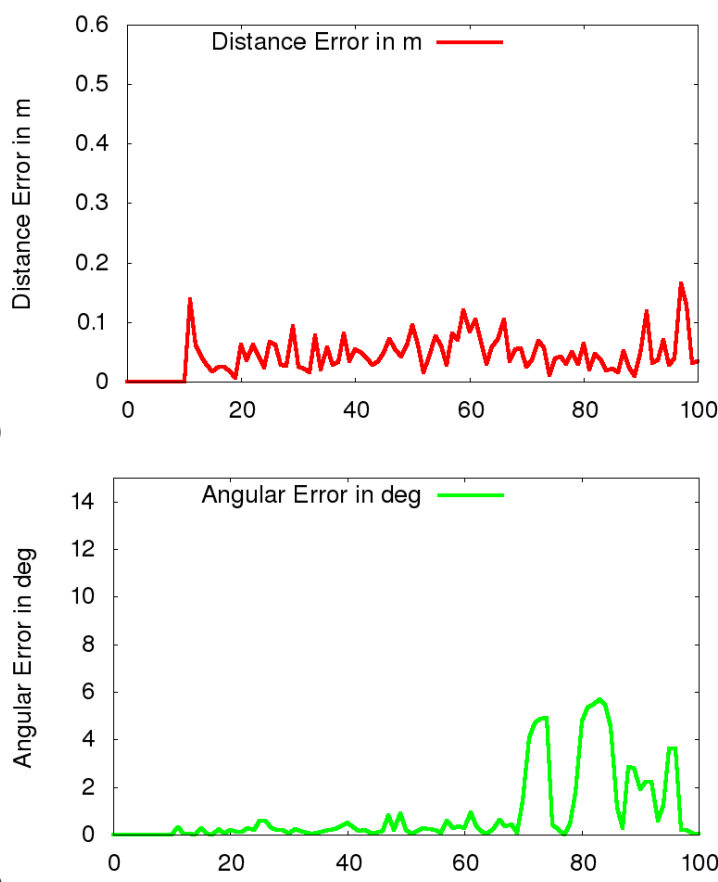

Figure 10: Translational (a) and rotational (b) errors of laserbased trajectory (100 3D scans) compared to MoCap trajectory.

\section{CONCLUSION}

We designed a small and lightweight continuously rotating 3D laser scanner that is particularly well suited for the use in lightweight UAVs. The sensor allows for measuring distances of up to $30 \mathrm{~m}$ in almost directions with a minimal blind spot. For each light pulse, up to three echoes are reported which is advantageous in case of transparent material, vegetation or edges of buildings.

We use the sensor to perceive obstacles in the vehicle's environment by building a grid-based obstacle map. We estimate the motion of our multicopter by registering 3D laser scans with the map.

We compared the laser-based ego-motion estimate with groundtruth data from a motion capture system. Overall, we can build an accurate 3D obstacle map and can estimate the vehicle's trajectory by $3 \mathrm{D}$ scan registration.

We integrated the sensor in our UAV and conducted experiments with dynamical obstacles, showing that the UAV is able to omnidirectional perceive obstacles and to react on them (Nieuwenhuisen et al., 2013).

\section{ACKNOWLEDGMENTS}

This work has been supported partially by grant BE 2556/7-1 of German Research Foundation (DFG).

\section{REFERENCES}

Bachrach, A., He, R. and Roy, N., 2009. autonomous flight in unstructured and unknown indoor environments. In: European Micro Aerial Vehicle Conf (EMAV), pp. 1-8.

Besl, P. J. and McKay, N. D., 1992. A method for registration of 3-D shapes. IEEE Transactions on Pattern Analysis and Machine Intelligence 14, pp. 239-256.
Bouabdallah, S., Murrieri, P. and Siegwart, R., 2004. Design and control of an indoor micro quadrotor. In: Proc. of the IEEE Int. Conf. on Robotics and Automation.

Cover, H., Choudhury, S., Scherer, S. and Singh, S., 2013. Sparse tangential network (spartan): Motion planning for micro aerial vehicles. In: Proc. of the IEEE Int. Conf. on Robotics and Automation.

Grzonka, S., Grisetti, G. and Burgard, W., 2009. Towards a navigation system for autonomous indoor flying. In: Proc. of the IEEE Int. Conf. on Robotics and Automation, Kobe (Japan).

Grzonka, S., Grisetti, G. and Burgard, W., 2012. A fully autonomous indoor quadrotor. IEEE Trans. on Robotics 28(1), pp. 90100.

Montemerlo, M., Becker, J., Bhat, S., Dahlkamp, H., Dolgov, D., Ettinger, S., Haehnel, D., Hilden, T., Hoffmann, G., Huhnke, B., Johnston, D., Klumpp, S., Langer, D., Levandowski, A., Levinson, J., Marcil, J., Orenstein, D., Paefgen, J., Penny, I., Petrovskaya, A., Pflueger, M., Stanek, G., Stavens, D., Vogt, A. and Thrun, S., 2008. Junior: The stanford entry in the urban challenge. Journal of Field Robotics.

Mori, T. and Scherer, S., 2013. First results in detecting and avoiding frontal obstacles from a monocular camera for micro unmanned aerial vehicles. In: Proc. of the IEEE Int. Conf. on Robotics and Automation.

Nieuwenhuisen, M., Schadler, M. and Behnke, S., 2013. Predictive potential field-based collision avoidance for multicopters. In: Proc. of the 2nd Conference on Unmanned Aerial Vehicles in Geomatics (UAV-g).

Puls, T., Kemper, M., Kuke, R. and Hein, A., 2009. GPS-based position control and waypoint navigation system for quadrocopters. In: Proc. of the IEEE/RSJ Int. Conf. on Intelligent Robots and Systems.

Ross, S., Melik-Barkhudarov, N., Shankar, K. S., Wendel, A., Dey, D., Bagnell, J. A. and Hebert, M., 2013. Learning monocular reactive uav control in cluttered natural environments. In: Proc. of the IEEE Int. Conf. on Robotics and Automation.

Scherer, S., Rehder, J., Achar, S., Cover, H., Chambers, A. D., Nuske, S. T. and Singh, S., 2012. River mapping from a flying robot: state estimation, river detection, and obstacle mapping. Autonomous Robots 32(5), pp. $1-26$.

Shen, S., Michael, N. and Kumar, V., 2011. Autonomous multifloor indoor navigation with a computationally constrained micro aerial vehicle. In: Robotics and Automation (ICRA), 2011 IEEE International Conference on, pp. $2968-2969$.

Tomić, T., Schmid, K., Lutz, P., Domel, A., Kassecker, M., Mair, E., Grixa, I., Ruess, F., Suppa, M. and Burschka, D., 2012. Toward a fully autonomous UAV: Research platform for indoor and outdoor urban search and rescue. Robotics Automation Magazine, IEEE 19(3), pp. 46-56.

Urmson, C., Anhalt, J., Bae, H., Bagnell, J. A. D., Baker, C. R., Bittner, R. E., Brown, T., Clark, M. N., Darms, M., Demitrish, D., Dolan, J. M., Duggins, D., Ferguson, D., Galatali, T., Geyer, C. M., Gittleman, M., Harbaugh, S., Hebert, M., Howard, T., Kolski, S., Likhachev, M., Litkouhi, B., Kelly, A., McNaughton, M., Miller, N., Nickolaou, J., Peterson, K., Pilnick, B., Rajkumar, R., Rybski, P., Sadekar, V., Salesky, B., Seo, Y.-W., Singh, S., Snider, J. M., Struble, J. C., Stentz, A. T., Taylor , M., Whittaker, W. R. L., Wolkowicki, Z., Zhang, W. and Ziglar, J., 2008. Autonomous driving in urban environments: Boss and the urban challenge. Journal of Field Robotics Special Issue on the 2007 DARPA Urban Challenge, Part I 25(8), pp. 425-466. 


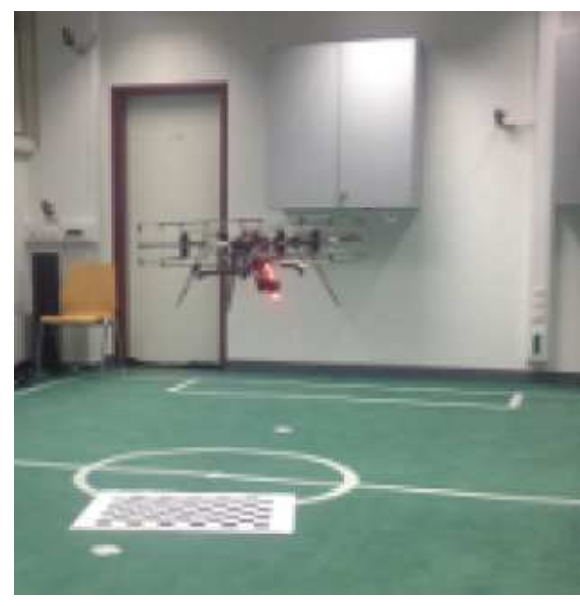

(a)

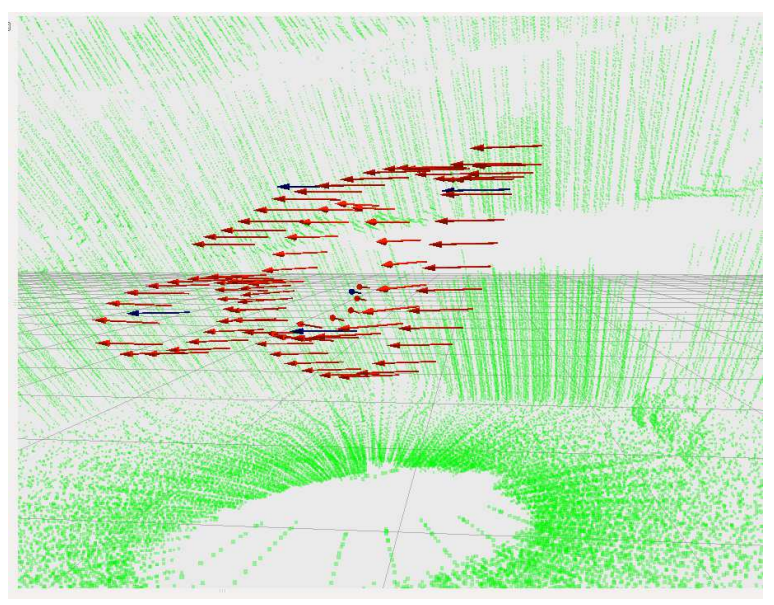

(b)

Figure 9: Evaluation of the scan registration in our motion capture system. (a) Setup. (b) Trajectory estimated by the algorithm (blue arrows) compared to the motion capture trajectory (red arrows).

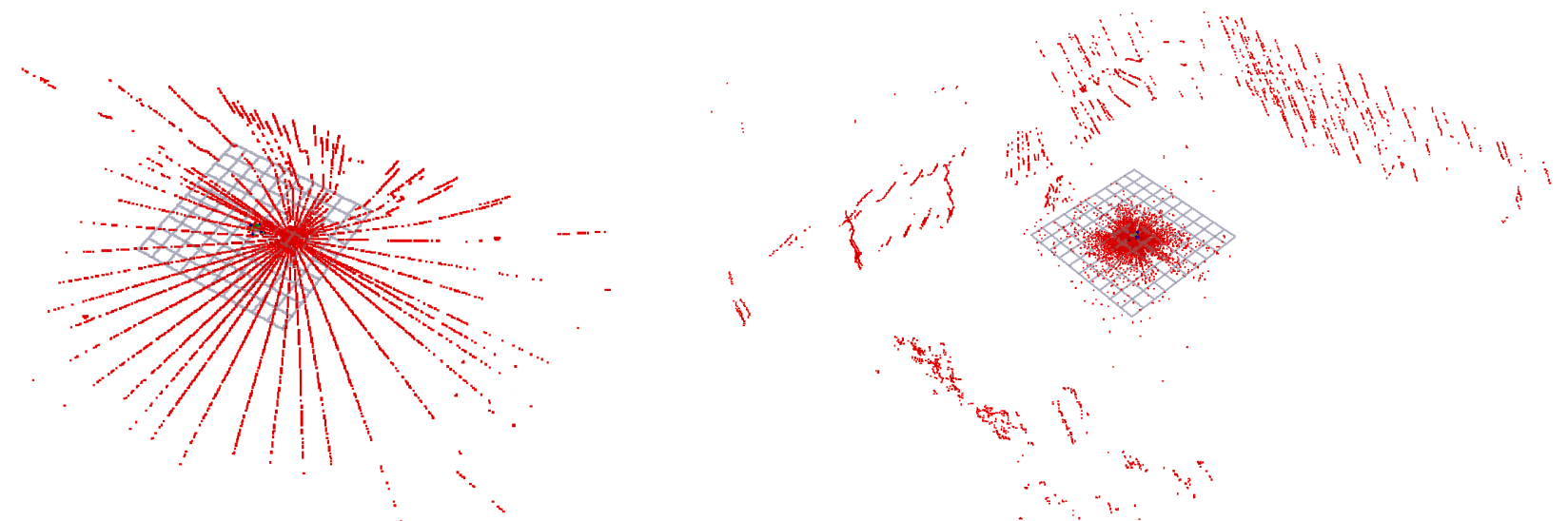

Figure 11: 3D scans acquired with our continuously rotating laser scanner in an outdoor environment during a flight. 